\title{
RETRACTED ARTICLE: Correlation of Promoter Methylation in the MGMT Gene with Glioma Risk and Prognosis: a Meta-Analysis
}

\author{
Xiang Dong $\cdot$ Rong-Yao Liu $\cdot$ Wei-Dong Chen
}

Received: 20 April 2014 / Accepted: 22 May 2014 / Published online: 17 August 2015

(C) Springer Science+Business Media New York 2014

The Publisher and Editor retract this article in accordance with the recommendations of the Committee on Publication Ethics (COPE). After a thorough investigation we have strong reason to believe that the peer review process was compromised.

The original article was published online on June 10, 2014.

X. Dong · R.-Y. Liu

Department of Neurology, First Hospital of Dalian Medical

University, Dalian 116011, People's Republic of China

W.-D. Chen $(\bowtie)$

Department of Neurosurgery, First Hospital of Dalian Medical

University, Zhongshan Road 222, Xigang District, Dalian 116011,

People's Republic of China

e-mail: chenweidong419@126.com 